\title{
Permeability and effective thermal conductivity of bisized porous media
}

\author{
Ricardo P. Dias ${ }^{\mathrm{a}, *}$, Carla S. Fernandes ${ }^{\mathrm{b}, 1}$, Manuel Mota ${ }^{\mathrm{c}, 2}$, \\ José A. Teixeira ${ }^{\mathrm{c}, 2}$, Alexander Yelshin ${ }^{\mathrm{c}, 2}$ \\ ${ }^{a}$ Departamento de Tecnologia Química, Escola Superior de Tecnologia e de Gestão, Instituto Politécnico de Bragança, Campus de Santa Apolónia, \\ Apartado 134, 5301-857 Bragança, Portugal \\ bepartamento de Matemática, Escola Superior de Tecnologia e de Gestão, Instituto Politécnico de Bragança, Campus de Santa Apolónia, \\ Apartado 134, 5301-857 Bragança, Portugal \\ ${ }^{\mathrm{c}}$ Centro de Eng. Biológica, Universidade do Minho, Campus de Gualtar, 4710-057 Braga, Portugal
}

Received 23 December 2005

Available online 22 January 2007

\begin{abstract}
In the region of minimum porosity of particulate binary mixtures, heat exchange and permeability were found to be higher than the ones obtained with a mono-size packing built with the same small size particles used in the binary packing. This effect was noticed in the range of the particles size ratio $0.1-1.0$.

The obtained improvement on thermal performance is related to the increase of effective thermal conductivity (ETC) in the binary packing and to the increase in transversal thermal dispersion due to the porosity decrease and tortuosity increase.

Permeability can increase by a factor of two, if the size ratio between small and large spheres of a loose packing stays in the range $0.3-$ 0.5 .

(C) 2006 Elsevier Ltd. All rights reserved.
\end{abstract}

Keywords: Fixed bed; Binary particulate mixtures; Permeability; Effective thermal conductivity

\section{Introduction}

Fixed beds are characterized by very good thermal performances, due to the high specific surface area and the convective heat transfer coefficients, and by poor hydraulic performances as a consequence of the low porosity [1].

Studies with high porosity metallic foams have been reported $[2,3]$ to overcome the large pressure drops of granular fixed beds, having the referred foams greater ETC than granular beds. This happens because in the latter the solid-solid contact thermal resistance effects are higher than in metallic foams. The same reason explains the

\footnotetext{
* Corresponding author. Tel.: +351 273303150; fax: +351 273313051.

E-mail address: ricardod@ipb.pt (R.P. Dias).

${ }^{1}$ Tel.: +351 273303127; fax: +351 273313051

${ }^{2}$ Tel.: +351 253604405; fax: +351 253678986 .
}

higher ETC from sintered porous media when compared with that from non-sintered material.

ETC ( or $K_{\mathrm{e}}$ ) of fluid saturated porous media can be experimentally determined by different techniques - in steady state [4] or transient state conditions [5,6], the latter presuming the existence of local thermal equilibrium between the fluid phase and the discontinuous phase $[7,8]$.

Solid-solid heat conduction through the finite contact area [9] between spherical particles becomes the main mechanism of heat transfer when the ratio between the solid and fluid conductivities, $K_{\mathrm{s}} / K_{\mathrm{f}}$, is higher than $10^{3}$. This seems to be the reason for the deviation of the Zehner and Schlünder [10] model from experimental measurements, since it is based on the assumption of contact points between particles and not on a finite contact area [11]. For values of $K_{\mathrm{s}} / K_{\mathrm{f}}<10^{3}$, conduction through the finite contact area loses importance and other mechanisms control the amount of transferred heat, such as the 


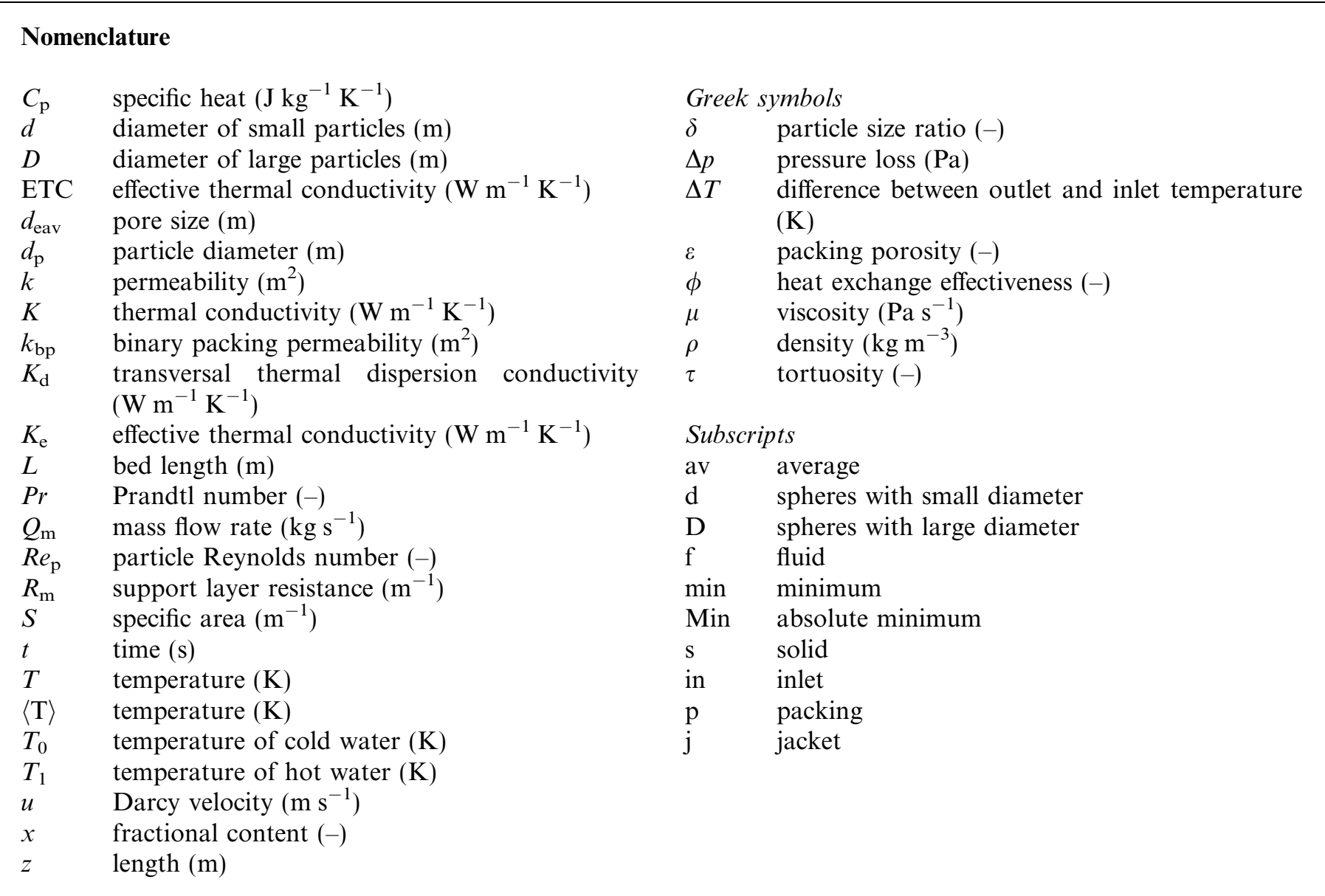

solid-fluid-solid conduction, resulting in a good agreement between experimental data and the Zehner and Schlünder model.

A particle-particle fraction contact area was introduced by Nozad et al. [5] on their 2-D unit cell, as well as Hsu et al. [12] on their in-line 3-D touching cubes model, and a good agreement was found with experimental data on a broad range of the ratio $K_{\mathrm{s}} / K_{\mathrm{f}}$.

For mono-size random packed beds, depending on the packing method, loose and dense packing (that present different standard coordination numbers) can be obtained $[13,14]$. The porosity of particle binary mixtures of spheres with different sizes depends on the fractional content, $x_{\mathrm{D}}$, of spheres with larger diameter, $D$, and on the mono-size components porosities and particle size ratio between them [15].

In particulate binary mixtures of spheres with different diameters, minimum porosity, $\varepsilon_{\min }$, is observed with a fractional content $x_{\mathrm{D}_{\min }}$ around 0.7 , for different particle size ratios, $\delta=d / D$. For the extreme case $\delta \rightarrow 0$ and using a linear mixture model [16], we have:

$x_{\mathrm{D}_{\min }}=\left(1-\varepsilon_{\mathrm{D}}\right) /\left(1-\varepsilon_{\mathrm{d}} \varepsilon_{\mathrm{D}}\right)$

where $\varepsilon_{\mathrm{d}}$ and $\varepsilon_{\mathrm{D}}$ are the mono-size porosities of the fractions with smaller diameter, $d$, and larger diameter, $D$, respectively, and where the porosities in the approximate range $0.36-0.41$ vary, depending on the packing method. The absolute minimum porosity, $\varepsilon_{\mathrm{Min}}$, is reached when $\delta \rightarrow 0$ and it is given by $\varepsilon_{\text {Min }}=\varepsilon_{\mathrm{d}} \cdot \varepsilon_{\mathrm{D}}$.

The main part of the minimum experimental binary packing porosity values, $\varepsilon_{\min }$, is located within the bounds defined by Eqs. (2) and (3), but these bounds might vary slightly, due to $\varepsilon_{\mathrm{d}}$ and $\varepsilon_{\mathrm{D}}$ dependence on packing conditions. The Liu and $\mathrm{Ha}$ [17] model describes $\varepsilon_{\min }$ well for dense packing:

$\varepsilon_{\min }=\varepsilon_{\text {Min }}+\left(\varepsilon_{\mathrm{d}}-\varepsilon_{\text {Min }}\right) \exp [0.25(1-1 / \delta)]$

whereas, for a loose packing, $\varepsilon_{\min }$ may be described by [16]:

$\varepsilon_{\min }=\varepsilon_{\mathrm{d}}\left(1-x_{\mathrm{D}_{\min }}\right) \exp \left(1.2264 x_{\mathrm{D}_{\min }}^{1 / \sqrt{\delta}}\right) /\left(1-\varepsilon_{\mathrm{d}} x_{\mathrm{D}_{\min }}\right)$.

Besides ETC, porosity affects the packing permeability $k$ $[14,18-20]$ :

$k=\frac{\varepsilon^{3} d_{\mathrm{p}}^{2}}{72 \tau^{2}(1-\varepsilon)^{2}}$

where $\tau$ is the pore tortuosity [21]. For particulate binary mixtures, the particle diameter $d_{\mathrm{p}}$ is calculated by the average, $d_{\mathrm{av}}$ :

$d_{\mathrm{av}}=\left(\frac{x_{\mathrm{D}}}{D}+\frac{1-x_{\mathrm{D}}}{d}\right)^{-1}$. 
Pore diameter, $d_{\text {eav }}$, and specific area, $S$, of the particulate binary mixtures are related to the particle size, being the former $d_{\mathrm{eav}}=2 d_{\mathrm{av}} \varepsilon / 3(1-\varepsilon)$ and the latter $S=6 / d_{\mathrm{av}}$. A wide range of average pore size on binary mixtures may be observed, depending on the fractional content.

Tortuosity is often modelled by the simple function:

$\tau=(1 / \varepsilon)^{n}$

where $n=0.4$ [20], 0.5 [22], or varies in the range $0.4-0.5$ [14], depending on the packing method and the packing composition.

$\mathrm{Du}$ et al. [23] deduced a correlation that translates the increase of transversal thermal dispersion conductivity, $K_{\mathrm{d}}$, with the tortuosity of a packed bed. They explained the decrease of $K_{\mathrm{d}}$ near the wall mainly by the decrease of tortuosity and increase of porosity at that region:

$\frac{K_{\mathrm{d}}}{K_{\mathrm{f}}}=D_{\mathrm{f}}(\tau-1)\left(1-\frac{\varepsilon}{\tau}\right) R e_{\mathrm{p}} P r_{\mathrm{f}}$

where $D_{\mathrm{f}}$ is an empirical constant assumed to be 0.35 , being $R e_{\mathrm{p}}$ the particle Reynolds number and $P r_{\mathrm{f}}$ the Prandlt number.

The aim of the current work is to compare the permeability and ETC from fixed beds containing glass spheres of uniform size with the permeability and ETC of fixed beds containing a mixture of glass spheres with different sizes (binary mixtures). The binary mixtures were built in the region of minimum porosity, applying a reproducible packing procedure developed in past investigations [24]. Forced convection is analysed using the concept of heat exchange effectiveness.

\section{Materials and methods}

The soda-lime glass spheres used on ETC, the permeability and the forced convection experiments were obtained from Sigmund Lindner and they had the following diameters: $1.9 \times 10^{-5} \mathrm{~m}, 1.15 \times 10^{-4} \mathrm{~m}, 1.5 \times 10^{-4} \mathrm{~m}$, $3.375 \times 10^{-4} \mathrm{~m}, 8.75 \times 10^{-4} \mathrm{~m}, 1.125 \times 10^{-3} \mathrm{~m}, 2 \times 10^{-3}$ $\mathrm{m}, 3 \times 10^{-3} \mathrm{~m}, 4 \times 10^{-3} \mathrm{~m}, 5 \times 10^{-3} \mathrm{~m}$ and $6 \times 10^{-3} \mathrm{~m}$.

ETC was determined in transient state using the Nozad et al. [6] procedure (Fig. 1). A prismatic transparent acrylic column with $0.05 \mathrm{~m}$ inner side and $0.2 \mathrm{~m}$ length was used. The walls were thermally insulated by polyurethane foam moulded for the prismatic column. Two copper plates with $1 \mathrm{~mm}$ thick were inserted on the top and bottom of the cell.

After achieving a uniform temperature of $293.15 \mathrm{~K}$ in the packing $\left(T_{0}\right)$, constant temperatures of $293.15 \mathrm{~K}\left(T_{0}\right)$ and $313.15 \mathrm{~K}\left(T_{1}\right)$ were imposed to the bottom and top of the packing by two thermostatic baths. Temperatures, $\langle T\rangle$, along the length, $z$, of the packing were recorded at different times, $t$, by six iron-constant thermocouples connected to a multi-channel microprocessor device. Assuming local thermal equilibrium between solid and fluid phases, the effective thermal diffusivity, $\alpha$, was estimated by [6]:

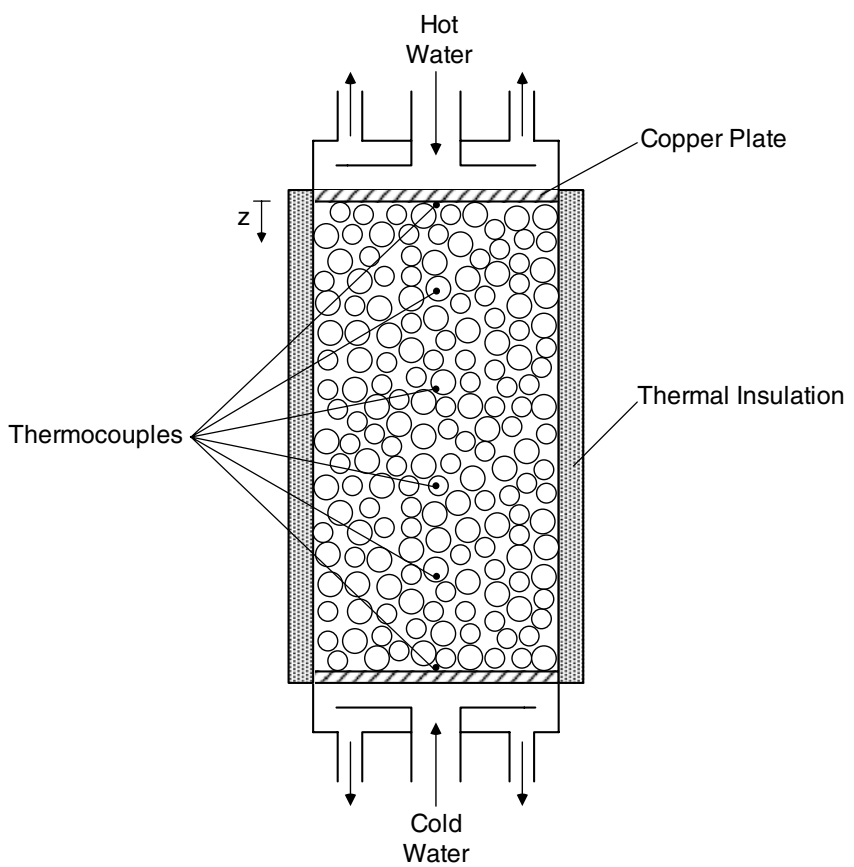

Fig. 1. Cell used in the effective thermal diffusivity measurements.

$\operatorname{erf}^{-1}\left(\frac{T_{1}-\langle T\rangle}{T_{1}-T_{0}}\right)=\frac{1}{\sqrt{\alpha}}\left(\frac{z}{2 \sqrt{t}}\right)$

The effective thermal conductivity could be calculated by measuring the porosity through the density method, and taking in account Eqs. (9) and (10) [11]:

$\alpha=K_{\mathrm{e}} /\left(\rho C_{\mathrm{p}}\right)_{\mathrm{av}}$

$\left(\rho C_{\mathrm{p}}\right)_{\mathrm{av}}=\rho_{\mathrm{f}} C_{\mathrm{pf}} \varepsilon+\rho_{\mathrm{s}} C_{\mathrm{ps}}(1-\varepsilon)$

where $\rho_{\mathrm{f}}$ and $\rho_{\mathrm{s}}$ are the fluid and solid density, and $C_{\mathrm{pf}}$ and $C_{\mathrm{ps}}$ are the fluid and solid specific heats, respectively. At a temperature of $303.15 \mathrm{~K}$, these parameters are equal to $1260 \mathrm{~kg} \mathrm{~m}^{-3}, 2500 \mathrm{~kg} \mathrm{~m}^{-3}, 2386 \mathrm{~J} \mathrm{~kg}^{-1} \mathrm{~K}^{-1}$ and 846 $\mathrm{J} \mathrm{kg}^{-1} \mathrm{~K}^{-1}$, respectively. The fluid and solid were glycerol and soda-lime glass in all ETC experiments. At $303.15 \mathrm{~K}$, the thermal conductivity of the fluid, $K_{\mathrm{f}}$, and of the solid, $K_{\mathrm{s}}$, were $0.292 \mathrm{~W} \mathrm{~m}^{-1} \mathrm{~K}^{-1}$ and $1.06 \mathrm{~W} \mathrm{~m}^{-1} \mathrm{~K}^{-1}$, respectively, being the physical data for the glass spheres obtained from Sigmund Lindner.

ETC was determined in mono-size packing beds, i.e. packings containing glass spheres of uniform size, and in binary packing beds, i.e. packing containing glass spheres with different sizes, saturated in all cases with glycerol. A method to obtain controlled binary packing beds, based on the application of a previous investigation, was applied. A water-glycerol solution was used as a binder between the different sized particles and the uniform distribution of the different size spheres within the packing was checked by image analysis [24]. The volume of glycerol used to saturate the mono-size and binary packing beds was calculated according to the binary packing porosity model from Mota et al. [20], for $\delta$ between 0.1 and 1 , and according to the model from Dias et al. [16], for $\delta$ lower than 0.1. 


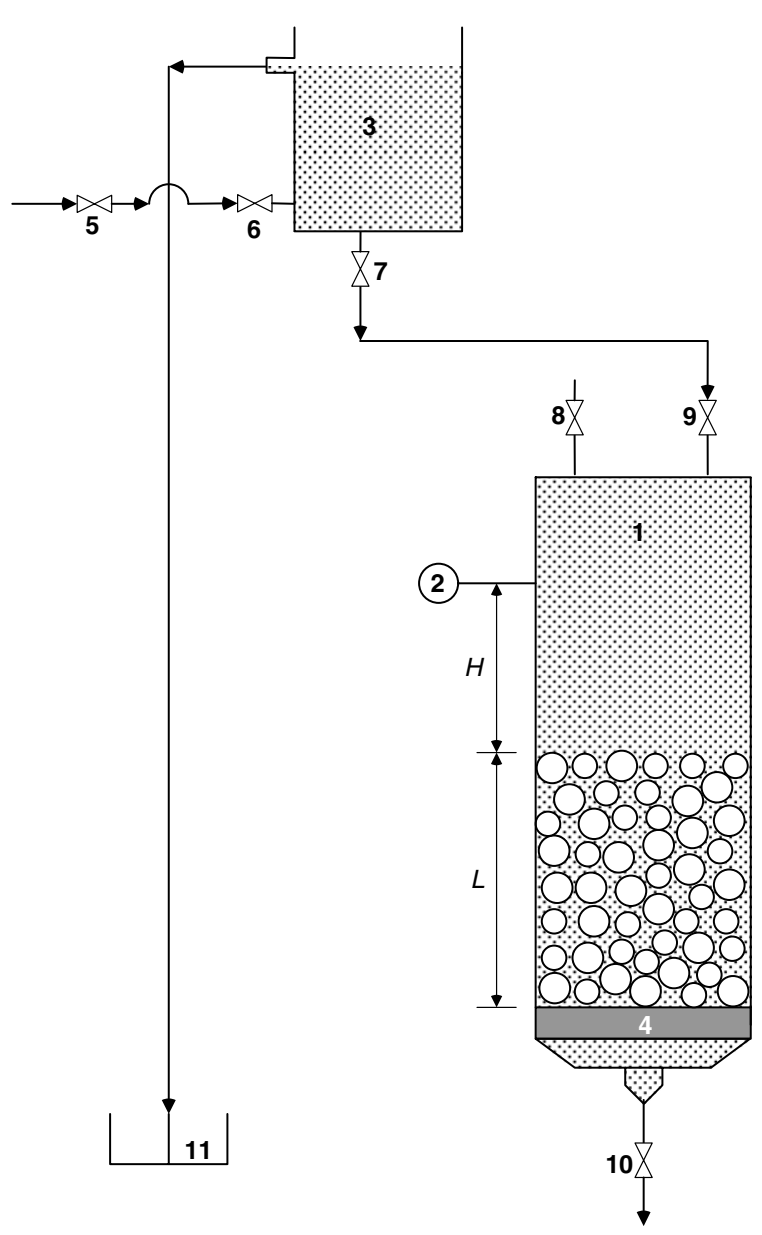

Fig. 2. Experimental setup used in the permeability measurements: $1-$ column; 2 - pressure gauge; 3 - tank with constant level of water; 4 - layer support; 5 to 10 - valves; 11 - drain.

The permeability of the constructed binary packing beds was determined by Eq. (11), measuring the flow rate at a fixed pressure [20] (Fig. 2).

$k=\frac{L}{\left(\frac{\Delta p}{\mu u}-R_{\mathrm{m}}\right)}$.

In Eq. (11), $\Delta p$ represents the pressure loss induced by the bed with length $L, R_{\mathrm{m}}$ the support layer resistance, $u$ the Darcy velocity and $\mu$ the water viscosity. The resistance $R_{\mathrm{m}}$ was determined in an experiment without bed $(L=0)$. The pressure on the top of the bed was estimated by the value observed on the pressure gauge and the height of water $H$ (Fig. 2). The cross-sectional area of the prismatic column and the volumetric flow rate obtained at the outlet of the experimental setup allowed the calculation of $u$.

The transparent acrylic square column used in experiments had $0.08 \mathrm{~m}$ on the inner side and $0.4 \mathrm{~m}$ length. A square wire cloth from Haver \& Boecker with aperture width $3.2 \times 10^{-5} \mathrm{~m}$ was used as a layer support. The packing length varied in all experiments between $0.1 \mathrm{~m}$ and $0.15 \mathrm{~m}$.
Forced convection was studied with the help of a borosilicate glass piston column from Omnifit having $0.025 \mathrm{~m}$ inner diameter and $0.25 \mathrm{~m}$ length. Using a thermostatic bath, the temperature of the water flowing in the jacket was $333.15 \mathrm{~K}$ at the inlet and $332.85-333.05 \mathrm{~K}$ at the outlet, in all the experiments. The temperatures of the water at the inlet and outlet of the column containing the packing were recorded, after reaching a steady state, at different mass flow rates imposed by an Ismatec Reglo peristaltic pump.

\section{Results and discussion}

Working on the region of minimum porosity, $\varepsilon_{\min }$, several glycerol saturated binary mixtures were constructed. The binary mixtures had a fractional content $x_{\mathrm{D}}$ of 0.7 and the particle size ratio $\delta$ varied between 0.0186 and 1 . The ETC from the constructed binary mixtures was studied by determining the effective thermal diffusivity and the porosity (Fig. 3).

Experimental thermal diffusivity data was fitted with the following relation:

$\alpha=4.550 \exp (-1.618 \varepsilon) \times 10^{-7}$

with a correlation coefficient $R^{2}=0.992$.

For the theoretical limit case $\varepsilon \rightarrow 0$, not achievable with particulate binary mixtures, Eq. (12) predicts a soda-lime glass thermal diffusivity, $\alpha_{\mathrm{s}}$, of $4.550 \times 10^{-7} \mathrm{~m}^{2} \mathrm{~s}^{-1}$, being the physical value $5.012 \times 10^{-7} \mathrm{~m}^{2} \mathrm{~s}^{-1}$, i.e. a $9.2 \%$ deviation.

ETC increased $28 \%$ with a change in porosity from 0.395 (mono-size packing) to 0.198 (binary packing with particle size ratio of 0.0186) (Fig. 4). A good agreement between the experimental data, for mono-size and binary packing, and the models described by Zehner and Schlünder, by Hsu et al. and by Krupiczka [10,12,25] was observed.

Experimental dependence of the minimum packing porosity, $\varepsilon_{\min }$, on $\delta$ is shown in Fig. 5 together with the

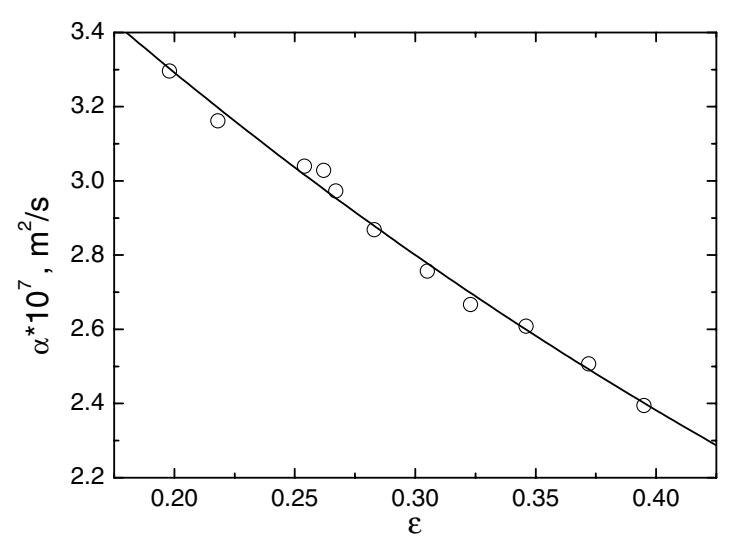

Fig. 3. Effective thermal diffusivity $\alpha$ from packing (mono-sized and binary) beds of glass beads with different porosity: circles - experimental data; line - Eq. (12). 


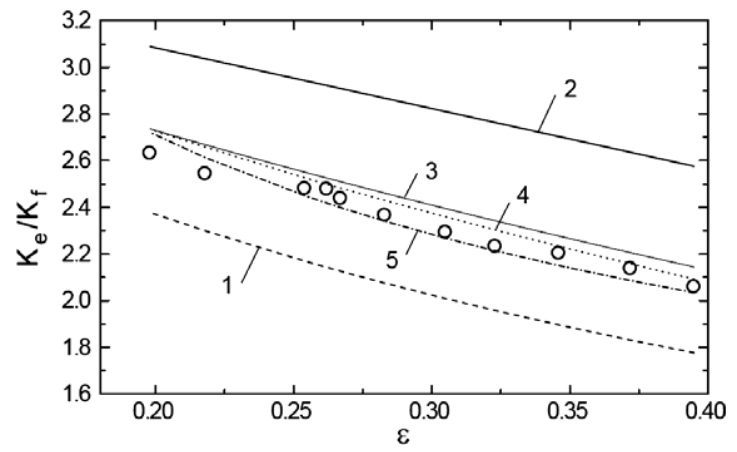

Fig. 4. Dependence of the ratio between ETC, $K_{\mathrm{e}}$, and fluid thermal conductivity on the packing porosity $\varepsilon$ : circles represent the experimental data; 1 - series-layer model; 2 - parallel-layer model; 3 - model [10]; 4 model with a particle-particle fraction contact area 0.13 [12]; 5 - model [25].

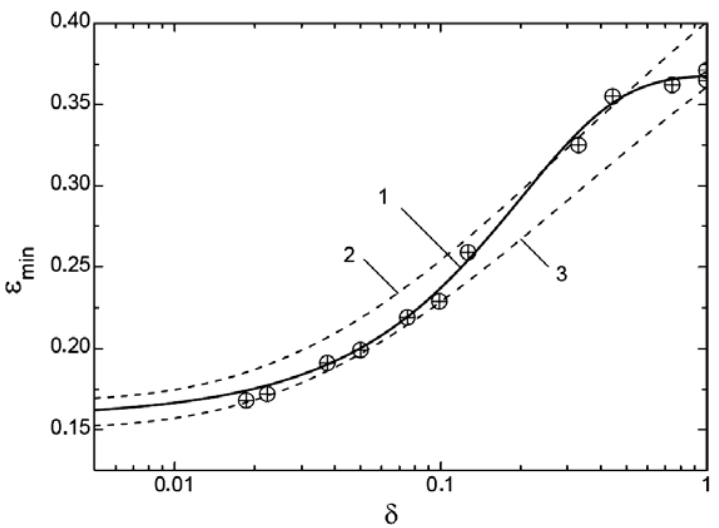

Fig. 5. Dependence of $\varepsilon_{\min }$ on the particle size ratio $\delta$. Points experimental data. Curve: 1 - fitting function, Eq. (13); curves 2 and 3 loose packing bounds, Eq. (3) for $x_{\mathrm{D}_{\min }}=0.7$ and $\varepsilon_{\mathrm{d}}=0.41$ and 0.38 , respectively.

loose packing model, Eq. (3), calculated for $x_{\mathrm{D}_{\min }}=0.7$ and $\varepsilon_{\mathrm{d}}=0.41$ and 0.38 . Obtained experimental data are on the range of the loose packing porosity. However, the different underlying mechanisms at high $\delta$ (exclusion) and small $\delta$ (linear-mixing) need an additional fitting procedure, provided by Eq. (13)

$\varepsilon_{\min }=0.368-0.615 /\{1+\exp [(\delta+0.1) / 0.153]\}$.

Current experiments, as well as previous investigations $[14,20]$, have shown that the packing composition content on binary mixtures, $x_{\mathrm{D}}$, where the minimum porosity is achieved, does not coincide with the $x_{\mathrm{D}}$, where minimum permeability is reached. Porosity and average pore size, dependent on particle diameter, interplay and explain this observation.

Using Eqs. (4)-(6) at a fractional content $x_{\mathrm{D}_{\min }}$ (Eq. (1)), correspondent to the region of minimum porosity $\varepsilon_{\min }$, the dependence of the dimensionless ratio $k_{\mathrm{bp}} / k_{\mathrm{d}}$ on $\delta$ is represented by the expression:

$\frac{k_{\mathrm{bp}}}{k_{\mathrm{d}}}=\left(\frac{\left(1-\varepsilon_{\mathrm{d}}\right)}{\left(1-\varepsilon_{\min }\right)}\right)^{2}\left(\frac{\varepsilon_{\min }}{\varepsilon_{\mathrm{d}}}\right)^{3+2 n}\left(\frac{1}{\left(\delta x_{\mathrm{D}}\right)+\left(1-x_{\mathrm{D}}\right)}\right)^{2}$.
In Eq. (14), $k_{\mathrm{bp}}$ stands for binary packing permeability, the packing that contains spheres with diameter $D\left(x_{\mathrm{D}}=0.7\right)$ and spheres with diameter $d$. The permeability of the mono-size packing of spheres with diameter $d$ is represented by $k_{\mathrm{d}}$.

Considering $\varepsilon_{\min }$ given by Eqs. (2) and (13) for dense and loose packing, respectively, it is possible to model the ratio $k_{\mathrm{bp}} / k_{\mathrm{d}}$ (Fig. 6). The $\delta$ region, where the permeability of binary packing, $k_{\mathrm{bp}}$, is higher than the permeability of mono-size packing of particles $d, k_{\mathrm{d}}$, is located in the range $0.1 \leqslant \delta<1.0$ for loose packing and $0.4 \leqslant \delta<1.0$ for dense packing.

As with porosity (Fig. 5), the normalised experimental permeability $k_{\mathrm{bp}} / k_{\mathrm{d}}$ (Fig. 6) also occupies the region between curves 2 and 3 corresponding to the limits of bounded loose packings. The shaded area in Fig. 6 corresponds to $k_{\mathrm{bp}} / k_{\mathrm{d}} \geqslant 1.0$, where the binary packing permeability for the loose packing is higher than the permeability of the mono-size loose packing of the smaller particles $d$.

By applying to Eq. (14), a more precise function (Eq. (13)) to describe the minimum porosity, a very good agreement with the experimental data is obtained. The room for a simultaneous increase of permeability and ETC is wider on loose packing and an increase in permeability by a factor of two was achieved for particle size ratios between 0.3 and 0.5 .

If a mono-size packing of spheres with diameter $D$ is being used on a certain application, the permeability of the related packing is greater than the permeability $k_{\mathrm{bp}}$ or $k_{\mathrm{d}}$. However, if a concomitant increase of ETC is desired, then a packing containing $30 \%$ of spheres with a diameter $D$ and $70 \%$ of spheres with a diameter higher than $D$ should be constructed. In a loose packing, a diameter higher than $D$ should provide a particle size ratio between

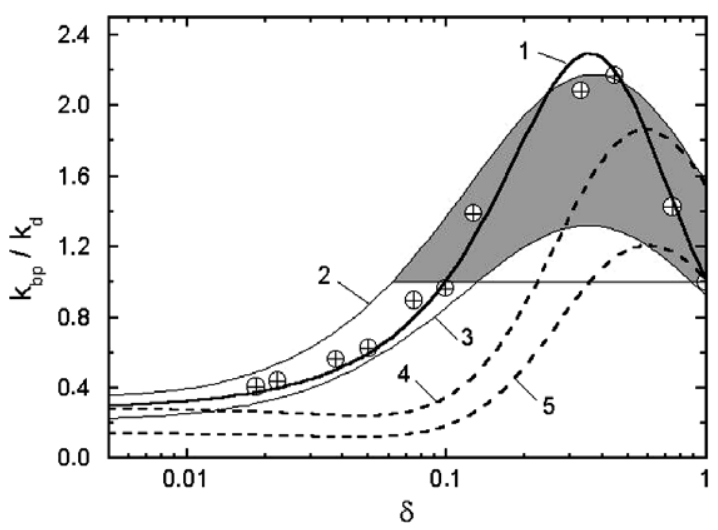

Fig. 6. Dependence of $k_{\mathrm{bp}} / k_{\mathrm{d}}$ (Eq. (14)) on the particles size ratio $\delta$ for $n=0.4$, using different models to describe $\varepsilon_{\min }$ : Points - experimental data; curve $1-\varepsilon_{\min }$ calculated by Eq. (13); curves 2 and $3-\varepsilon_{\min }$ calculated by Eq. (3) for $x_{\mathrm{D}_{\min }}=0.7$ when $\varepsilon_{\mathrm{d}}=0.41$ and 0.38 , respectively; curves 4 and $5-\varepsilon_{\min }$ calculated by Eq. (2) at $\varepsilon_{\mathrm{d}}=\varepsilon_{\mathrm{D}}=0.4$ and 0.368 , respectively. Considering loose packings, the shaded area corresponds to the zone where $k_{\mathrm{bp}} / k_{\mathrm{d}} \geqslant 1.0$. 
0.1 and 1, in order to obtain a simultaneous ETC and permeability increase.

In Fig. 7, the thermal performance of one binary packing is compared with the thermal performance of the mono-size packing of spheres with diameter $d$. The mono-size and binary packing had the same length and, respectively, the additional following characteristics: spheres with diameter $d=1.5 \times 10^{-4} \mathrm{~m}$ and porosity $\varepsilon_{\mathrm{d}}=$ $0.392,30 \%$ of spheres with $d=1.5 \times 10^{-4} \mathrm{~m}$ and $70 \%$ $\left(x_{\mathrm{D}}=0.7\right)$ with $D=1.125 \times 10^{-3} \mathrm{~m}(\delta=0.133)$ and $\varepsilon=$ 0.267 . For the size ratio 0.133 , the permeability increase over the mono-size packing is around $30-35 \%$ (Fig. 6). The temperature difference, $\Delta T$, between the inlet and outlet of the packed beds was measured at different mass flow rates $Q_{\mathrm{m}}$. Using the experimental $\Delta T$ values, the heat exchange effectiveness $\phi[1,26]$ was computed by Eq. (15):

$\phi=\Delta T /\left(T_{\mathrm{in}, \mathrm{j}}-T_{\mathrm{in}, \mathrm{p}}\right)$,

where $T_{\text {in.j }}$ and $T_{\text {in,p }}$ is the water inlet temperature in the jacket and packing, respectively.

Being that for the present mass flow rate in the jacket $\left(0.0805 \mathrm{~kg} \mathrm{~s}^{-1}\right)$ when $Q_{\mathrm{m}} \rightarrow 0$ the heat exchange effectiveness should tend to one, the following relations can be found for the mono-size packing (Eq. (16)), and for the binary packing (Eq. (17)):

$\phi=0.3+0.7 \exp \left(-Q_{\mathrm{m}} / 0.00068\right), \quad R^{2}=0.999$

$\phi=0.187+0.81 \exp \left(-Q_{\mathrm{m}} / 0.00103\right), \quad R^{2}=0.998$.

It can be observed in Fig. 7 that the heat exchange effectiveness of the binary packing, having a mixture of spheres with diameter $1.5 \times 10^{-4} \mathrm{~m}$ and $1.125 \times 10^{-3} \mathrm{~m}$, is higher than the heat exchange effectiveness of the mono-size packing, having spheres with diameter $1.5 \times 10^{-4} \mathrm{~m}$. The permeability of the related binary packing is also higher than the permeability of the mono-size packing (Fig. 6 for $\delta=0.133$ ).

Despite the mono-size packing of spheres with diameter $d$, having the largest specific superficial area, $6 / d$, a higher

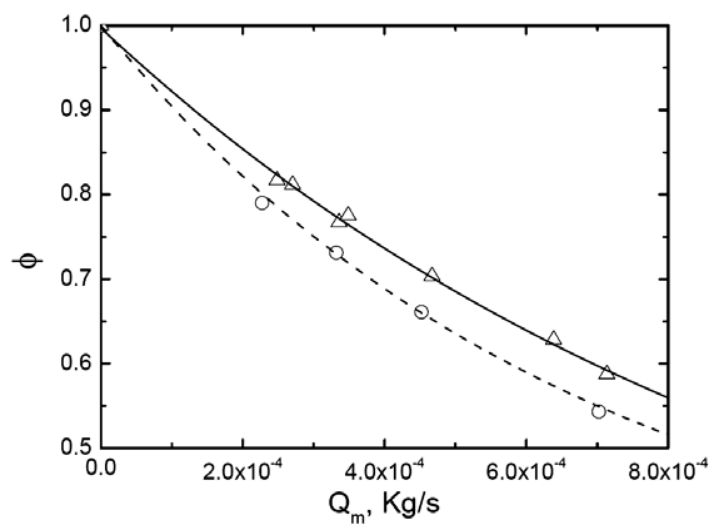

Fig. 7. Heat exchange effectiveness $\phi$ for flow in a mono-size packing of glass spheres with diameter $1.5 \times 10^{-4} \mathrm{~m}(\circ)$; and a binary packing with $x_{\mathrm{D}}=0.7$ and $\delta=0.133(\triangle)$. Curves: (---) Eq. (16); (-) Eq. (17). thermal efficiency was obtained through the binary mixture with specific superficial area $6 / d_{\mathrm{av}}$. The $7 \%$ increase [10] of ETC, in transition from the mono-size to the binary packing, and the thermal dispersion increase due to the tortuosity increase and porosity decrease Eq. (7) play a decisive role on the observed result. On the two packing types reported in Fig. 7, the term $(\tau-1)(1-\varepsilon / \tau)$ from Eq. (7) assumes a value of 0.59 and 0.33 on the binary packing and mono-size packing, respectively. The used tortuosity is given by Eq. (6) being $n=0.4[14,20]$.

Concerning permeability, the results from the current work with glass spheres can be safely extended to spheres of other rigid materials. The same happens with the conclusions suggested by Eq. (7) for thermal dispersion, since the increase on tortuosity and decrease of porosity associated to the use of binary mixtures of spheres with different sizes is independent from the used materials. The models available in the literature [12] consider the effect of the porosity and also of solids thermal conductivity on ETC, and show a good agreement with experimental data in a broad range of the ratio $K_{\mathrm{s}} / K_{\mathrm{f}}$ [8]. It is then reasonable to admit that ETC will increase with the decrease of porosity on the transition from mono-size to binary packing of spheres of other materials besides glass.

Together with the models available in the literature $[10,12]$, Eq. (3) or (13) allows the estimate of ETC from binary packing on the region of minimum porosity, for different particle size ratios, $\delta$. Lowering the porosity in this region (by the decrease of $\delta$ ), higher values of ETC can be obtained for a certain system solid/fluid. However, permeability (Fig. 6) will decrease with the decrease of the particle size ratio, if $\delta$ is located below 0.3 (in the loose packing case). Considering the porosity of the two packing types reported in Fig. 7, but a higher ratio $K_{\mathrm{s}} / K_{\mathrm{f}}$, a higher increment of ETC on the transition from the mono-size to binary packing can be obtained. As already referred, that ETC increment for the system glass/water $\left(K_{\mathrm{s}} / K_{\mathrm{f}} \cong 1.7\right)$ is close to $7 \%$. Considering the porosity of the two packing types reported in Fig. 7, this increment can reach a value of $75 \%$ [10] for a moderate value of $K_{\mathrm{s}} / K_{\mathrm{f}}=10^{3}$.

\section{Conclusions}

Particulate binary mixtures, especially in the case of loose packing, may give rise to a substantial improvement of the thermal-hydraulic performance associated to monosize packing.

It was shown by experimental data and different models available in the literature that it is possible to achieve a permeability enhancement with dense and loose packing. The behaviour of porosity on the region of minimum porosity of particulate binary mixtures explains the referred enhancement.

Heat exchange enhancement can be explained by the increase of ETC, due to the porosity reduction associated 
to binary mixtures construction, and by the increase on transversal thermal dispersion, due to the tortuosity increase and porosity decrease.

The current investigation can have practical hydraulic and thermal implications on chromatographic columns, filter beds, heat exchangers and chemical reactors. Further experiments with binary mixtures are needed in order to estimate convective heat transfer coefficients.

\section{Acknowledgement}

The authors thank FCT for the grant accorded to A. Yelshin. They also thank FCT for having provided the funds to perform this work through the project POCI/ EQU/58337/2004. This project was partially funded by FEDER.

\section{References}

[1] P.-X. Jiang, M.-H. Fan, G.-S. Si, Z.-P. Ren, Thermal-hydraulic performance of small scale micro-channel and porous-media heatexchangers, Int. J. Heat Mass Transfer 44 (2001) 1039-1051.

[2] J.W. Paek, B.H. Kang, S.Y. Kim, J.M. Hyun, Effective thermal conductivity and permeability of aluminium foam materials, Int. J. Thermophys. 21 (2000) 453-464.

[3] A. Bhattacharya, V.V. Calmidi, R.L. Mahajan, Thermophysical properties of hight porosity metal foams, Int. J. Heat Mass Transfer 45 (2001) 1017-1031.

[4] M. Gupta, J. Yang, C. Roy, Modelling the effective thermal conductivity in polydispersed bed systems: a unified approach using the linear packing theory and unit cell model, Can. J. Chem. Eng. 80 (2002) 830-839.

[5] I. Nozad, R.G. Carbonell, S. Whitaker, Heat conduction in multiphase systems. I. Theory and experiment for two-phase systems, Chem. Eng. Sci. 40 (1985) 843-855.

[6] I. Nozad, R.G. Carbonell, S. Whitaker, Heat conduction in multiphase systems. II. Experimental method and results for three-phase systems, Chem. Eng. Sci. 40 (1985) 857-863.

[7] M. Quintard, S. Whitaker, Local thermal equilibrium for transient heat conduction: theory and comparison with numerical experiments, Int. J. Heat Mass Transfer 38 (1995) 2779-2796.

[8] C.T. Hsu, Heat conduction in porous media, in: K. Vafai (Ed.), Handbook of Porous Media, Marcel Dekker, New York, 2000, pp. 170-200.
[9] C. Argento, D. Bouvard, Modeling the effective thermal conductivity of random packing of spheres through densification, Int. J. Heat Mass Transfer 39 (1996) 1343-1350.

[10] P. Zehner, E.U. Schlünder, Thermal conductivity of granular materials at moderate temperatures (in German), Chem. Ing. Tech. 42 (1970) 933-941.

[11] P. Cheng, C.T. Hsu, Heat conduction, in: D.B. Ingham, I. Pop (Eds.), Transport Phenomena in Porous Media, Pergamon, Oxford, 1998, pp. 57-76.

[12] C.T. Hsu, P. Cheng, K.W. Wong, A lumped parameter model for stagnant thermal conductivity of spatially periodic porous media, ASME J. Heat Transfer 117 (1995) 264-269.

[13] J.D. Bernal, J. Mason, Co-ordination of randomly packed spheres, Nature 188 (1960) 908-911.

[14] M. Mota, J.A. Teixeira, R. Dias, A. Yelshin, Effect of real particles packing with large size ratio on porosity and tortuosity of filter bed, in: Proceedings of 9th World Filtration Congress, New Orleans, AFS, 2004, Paper 316-3, pp. 1-30.

[15] R.P. Zou, A.B. Yu, Evaluation of the packing characteristics of mono-sized non-spherical particles, Powder Technol. 88 (1996) 71-79.

[16] R. Dias, J.A. Teixeira, M. Mota, A. Yelshin, Particulate binary mixtures: dependence of packing porosity on particle size ratio, Ind. Eng. Chem. Res. 43 (2004) 7912-7919.

[17] S. Liu, Z. Ha, Prediction of random packing limit for multimodal particle mixtures, Powder Technol. 126 (2002) 283-296.

[18] R. Ben Aim, P. Le Goff, P. Le Lec, La perméabilité de milieux poreux formés par empilement de mélanges binaires de grains sphériques, Powder Technol. 5 (1971) 51-60.

[19] M.J. MacDonald, C.-F. Chu, P.P. Guilloit, K.M. Ng, A generalized Blake-Kozeny equation for multisized spherical particles, AIChE J. 37 (1991) 1583-1588.

[20] M. Mota, J.A. Teixeira, A. Yelshin, Binary spherical particle mixed beds porosity and permeability relationship measurement, Trans. Filtration Soc. 1 (2001) 101-106.

[21] P.C. Carman, Fluid flow through granular beds, Trans. Inst. Chem. Eng. 15 (1937) 150-166.

[22] T.C. Zhang, P.L. Bishop, Evaluation of tortuosity factors and effective diffusivities in biofilms, Water Res. 28 (1994) 2279-2287.

[23] J. Du, X. Hu, W. Wu, B.-X. Wang, A thermal dispersion model for single phase flow in porous media, Heat Transfer - Asian Res. 32 (2003) 545-552.

[24] R. Dias, J.A. Teixeira, M. Mota, A. Yelshin, Preparation of controlled particulate mixtures with glass beads of different sizes, Separation Purif. Technol. 37 (2004) 69-80.

[25] R. Krupiczka, Analysis of thermal conductivity in granular materials, Int. Chem. Eng. 7 (1967) 122-144.

[26] S. Kakaç, H. Liu, Heat Exchangers: Selection, Rating, and Thermal Design, second ed., CRC Press, Boca Raton, 2002 (Chapter 2). 